\title{
Tailored sedation in critically ill COVID-19 patients based on lung damage, and pharmacokinetic and pharmacodynamic profiles
}

\author{
Alfredo Del Gaudio, Giuseppe Mincolelli ${ }^{1}$, Andreaserena Recchia ${ }^{1}$, Elvio De Blasio², \\ Maura C Tracey ${ }^{3}$, Marco Cascella ${ }^{4, *}$
}

\author{
${ }^{1}$ DSC Anestesia e Rianimazione 2, IRCCS \\ Casa Sollievo Della Sofferenza, 71013 \\ San Giovanni Rotondo (FG), Italy \\ ${ }^{2}$ Multidisciplinary Emergency Unit for \\ COVID-19 Campania, 80100 Naples, Italy \\ ${ }^{3}$ Scientific Directorate, Istituto Nazionale \\ Tumori "Fondazione G. Pascale"-IRCCS, \\ 80133 Naples, Italy \\ ${ }^{4}$ Division of Anesthesia and Pain \\ Medicine, Istituto Nazionale \\ Tumori-IRCCS-"Fondazione G. Pascale", \\ 80131 Naples, Italy
}

\section{*Correspondence}

m.cascella@istitutotumori.na.it

(Marco Cascella)

\begin{abstract}
In critically ill COVID-19 patients, proper management of sedation is an important issue. Therefore, for this purpose, several strategies and protocols have been proposed. In this paper, we illustrate an approach focused on lung damage, and both the pharmacokinetic and pharmacodynamic profiles of drugs used. In line with this, during high flow nasal (HFN), continuous positive airway pressure, or non-invasive ventilation, dexmedetomidine-based light sedation can be helpful for maintaining the respiratory driving and improving the patient comfort. A worsening in the respiratory clinical picture with mechanical ventilation may require deep sedation with the use of clonidine. The latter may reduce the hypnotic doses, allowing improved hemodynamic stability. When respiratory performance improves, dexmedetomidine can replace clonidine to reduce the time to extubation.
\end{abstract}

Keywords

COVID-19; Mechanical ventilation; Sedation; Propofol; Dexmedetomidine

\section{Introduction}

Since the main characteristic of COVID-19 is its evolutionary potential, understanding the correct pathophysiology of the disease is crucial in establishing the most appropriate treatment. Progression of COVID-19 pneumonia can lead to the development of a time-related disease spectrum within two main "phenotypes" regarding lung involvement. As Gattinoni et al. [1] proposed, the Type L phenotype is characterized by low elastance (i.e., high compliance), low ventilation-toperfusion ratio, low lung weight, and low recruitability. On the other hand, the Type $\mathrm{H}$ has high elastance, high right-toleft shunt, high lung weight, and high recruitability.

In patients with severe acute respiratory distress syndrome (ARDS), mechanical ventilation (MV) is aimed at improving gas exchange, oxygen transport, and tissue oxygenation, limiting ventilator-induced lung injury (VILI), potentially associated with prolonged ventilation.

Management of sedation represents an issue to be addressed and several strategies have been proposed [2,3]. These approaches can be based on the use of multiple drugs. In critically ill patients, intravenous sedatives such as propofol, remifentanil, $\alpha 2$-adrenergic receptor ( $\alpha 2$-AR) agonists, and benzodiazepines, or volatile agents such as sevoflurane, and isoflurane can be used. Since the serotonin system plays a key role in modulating the sleep/wake cycle, and in genesis of delirium [4], the anti-serotonin agent cyproheptadine should be considered for sedation in COVID-19 patients. Furthermore, at least theoretically, this drug could be helpful in reducing the risk of pulmonary fibrosis [5]. Nevertheless, according to the recent Pain, Agitation/Sedation, Delirium, Immobility and Sleep disruption (PADIS) guidelines [6], the use of strict protocols that are applicable for all patients is not recommended [3]. According to the stage of the disease, a patient can require different levels of sedation. If we consider Gattinoni's model valid, during the L phase, high flow nasal (HFN), continuous positive airway pressure (CPAP) or non-invasive ventilation (NIV) must be assured; although sedation is helpful for reducing stress, the maintenance of the respiratory drive is mandatory. Light sedation [Richmond Agitation-Sedation Scale (RASS) 0 to -1] is a key component of the multiinterventional approach that includes optimal analgesia and goal-directed minimal sedation. Probably, in this phase, the highly selective $\alpha 2$-AR agonist dexmedetomidine (DEX) may represent an optimal choice, because it allows the patient to adapt to HFN, CPAP or NIV, preserving optimal hemodynamic stability (by reducing cardiac work and stress), and maintaining the respiratory drive. Several investigations demonstrate the advantages of DEX in COVID-19 patients. Paternoster et al. [7], for example, used this sedative to allow awake pronation with helmet CPAP outside the ICU.

A further increase of the inflammatory response leads to the $\mathrm{H}$ phase where the respiratory picture declines. In this stage, the patient should be treated as severe ARDS; therapy includes higher positive end-expiratory pressure (PEEP), if compatible hemodynamically, and the use of prone positioning. The 
TAB L E 1. Pharmacokinetics and pharmacodynamics of clonidine and dexmedetomidine [9, 10].

Pharmacokinetics

\section{Clonidine}

Administered orally, it is well absorbed and exhibits a minor first pass effect.

Absorption and distribution

Peak plasma concentration reached in $1-3 \mathrm{hrs}$.

It is rapidly and extensively distributed to tissues and passes both the $\mathrm{BBB}$ and the placenta.

Plasma protein binding is $30-40 \%$.

Metabolism and elimination

The elimination half-life of clonidine ranged 6-20 hrs (up to $41 \mathrm{~h}$ in patients with severe renal dysfunction). About $70 \%$ of the administered dose is excreted in the urine, mainly in unchanged form $(40-60 \%)$. The major metabolite (p-hydroxyclonidine) is inactive.

Pharmacodynamics

The action is mainly carried out at the level of the CNS, with reduction of sympathetic tone and peripheral resistance, heart rhythm, and blood pressure. The renal plasma flow and glomerular filtration remain unchanged. Normal postural reflexes do not change.

Compared to dexmedetomidine, it has fewer sedative properties $(600$ times lower)

\section{Dexmedetomidine}

Intravenous administration

Two-compartment distribution model with rapid distribution phase and distribution half-life $(\mathrm{t} 1 / 2 \alpha)$ of approximately 6 minutes.

Clearance: $0.46-0.73 \mathrm{~L} / \mathrm{h} / \mathrm{kg}$

Plasma PK is similar after $>24 \mathrm{~h}$ infusion and is linear with no accumulation in treatments lasting up to 14 days (dose range 0.2-1.4 $\mu \mathrm{g} / \mathrm{kg} / \mathrm{h})$.

Plasma protein binding is $30-40 \%$.

It is eliminated primarily through hepatic metabolism (Nglucuronidation, N-methylation, and oxidation by cytochrome P450). The metabolites have negligible pharmacological activity.

Highly selective for $\alpha 2$-receptors with an $\alpha 2: \alpha 1$ ratio of $1620: 1$. Sympatholytic effect through inhibition of the release of norepinephrine. Sedative effects due to decrease in the discharge activity of the LCN. Cardiovascular effects depend on the dose; at lower infusion rates, the central effects predominate with a decrease in heart rate and blood pressure. At higher doses, there is peripheral vasoconstrictor effects with an increase in systemic vascular resistance and blood pressure.

Legend: BBB, blood-brain barrier; CNS, central nervous system; PK, pharmacokinetics; LCN, locus coeruleus norepinephrine. 
required invasive MV imposes deep/heavy sedation (RASS -3 to -5$)$ through the use of hypnotic and analgesic drugs. This degree of sedation is also required to avoid unintentional self-extubation and to guarantee the prone positiong when required. Although in this early phase of severe ARDS, the Rapid Practice Guidelines recommended the use of neuromuscular blocking agents (NMBAs) to optimize MV [2], strategies of analgesia/sedation for lung protective ventilation without NMBAs should be carefully considered to improve patient outcomes [3].

Deep sedation requirements are likely related to younger age, high respiratory drive, and intense inflammatory responses; this translates into the need to administer combinations of multiple agents [8]. Consequently, a multimodal patient-centered approach is required but the combination and dosage of drugs used must be appropriately calibrated [8]. In this context, in combination with propofoland with or without remifentanil-clonidine can replace DEX. Since compared to DEX, clonidine has fewer sedative properties (600 times lower), it can reduce the hypnotic dose, guaranteeing improved hemodynamic stability, and delirium prevention [9, 10] (Table 1).

Thus, the cornerstone in treatment becomes drug synergism that improves the therapeutic window and avoids side effects [11].

Subsequently, as respiratory mechanics improve, it is necessary to reduce the level of sedation [12]. In this state, a strategy of light sedation can reduce the time to extubation with beneficial effects on sedation-induced complications. Since sedatives with drug accumulation effects should be avoided, DEX alone appears to be the best choice, shortening the duration of MV and improving patient comfort [13]. This drug could also exert anti-inflammatory effects [14]. Zhao et al. [15] for instance, underlined the potential effect of DEX in ameliorating the SARS-CoV-2-induced cytokine storm [16]. DEX also has important anti-delirium [17] and opioid-benzodiazepinesparing properties [18].

\section{Proposed strategy}

We propose an approach to sedation which is tailored to the degree of lung impairment, pharmacokinetics (PK) and pharmacodynamics (PD) drug profiles. The transition from minimal sedation to deep/heavy sedation, and vice versa, can be performed in line with the different phenotypes of COVID pneumonia (Type L and Type H). Consequently, different sedatives can be adopted according to their PK and PD properties. In brief, the Type L lung phenotype may require a DEX-based light/moderate sedation for maintaining the respiratory drive and improving patient comfort. A decline in the respiratory picture featuring the Type $\mathrm{H}$, is addressed through MV and deep sedation. In this stage, clonidine can be preferred to DEX because it has fewer sedative properties (600 times lower), and can reduce the hypnotic dose. Finally, when the respiratory performance improves, restoring of the DEX-based light sedation can reduce the time to extubation with beneficial effects on sedation-induced complications (Fig. 1). This 'dynamic' strategy can also allow synchronization the level of ventilator support to the target of sedation.
The PADIS study group has already proposed a dynamic strategy [3]. Their pathway involved a four-level model of sedation including the minimal, mild (RASS -1 to +1 ), moderate (RASS -2 to -3 ), and deep (RASS -3 to -5 ) level. It provided the use of analgesics or low-dose sedatives as the first step, and propofol with or without DEX, or in selected cases (e.g., those who are refractory to propofol or DEX) benzodiazepines, anti-psychotic agents, or volatile anesthetics. However, in this model the drugs are used at different dosages and based on patient symptoms and not in agreement with the PK/PD properties.

\section{Open issues}

Several open issues remain to be solved. For instance, what Czepiel et al. [19] reported about the occurrence of DEXassociated hyperpyrexia in three critically ill COVID-19 patients must be verified.

A particularly important aspect concerns the medication of choice for sedation. This decision is usually made according to the PK/PD features which include, for instance, the context-sensitive half-life analgesic properties, and potential respiratory depression. Nevertheless, $\mathrm{PK} / \mathrm{PD}$ is only one part of those characteristics. Furthermore, in critically ill patients, the PK/PD profile will change due to various reasons such as renal function, drug interaction, and acute tolerance.

Nevertheless, the major concern regards the inclusion of the Type L and Type H phenotypes for COVID-19 pneumonia and the evidence to support these. To date, pieces of evidence are not enough for sufficiently adding this phenomenon to the chapter of the pathophysiology of the disease. Nevertheless, pending further research results, we are highlighting this part of the literature on the issue, and our experience in the field of critically ill patient sedation and COVID-19 management.

\section{Conclusions}

In critically ill patients, the choice of the more accurate sedation strategy is a major issue. Although $\mathrm{PK} / \mathrm{PD}$ properties are helpful in planning an appropriate treatment regimen, additional factors, mostly related to patient co-morbidities and therapies administered, must necessarily be addressed. In the COVID-19 patients, additional difficulties arise. Some of them are linked to the different stages of the disease. We propose a dynamic approach to the sedation problem which is tailored to the degree of lung impairment.

\section{AUTHOR CONTRIBUTIONS}

ADG, GM and MC designed the study and collected the data. EDB drafted the manuscript. AR and MCT revised it critically for important intellectual contents. All authors approved the final version to be published.

\section{ETHICS APPROVAL AND CONSENT TO PARTICIPATE}

Not applicable. 


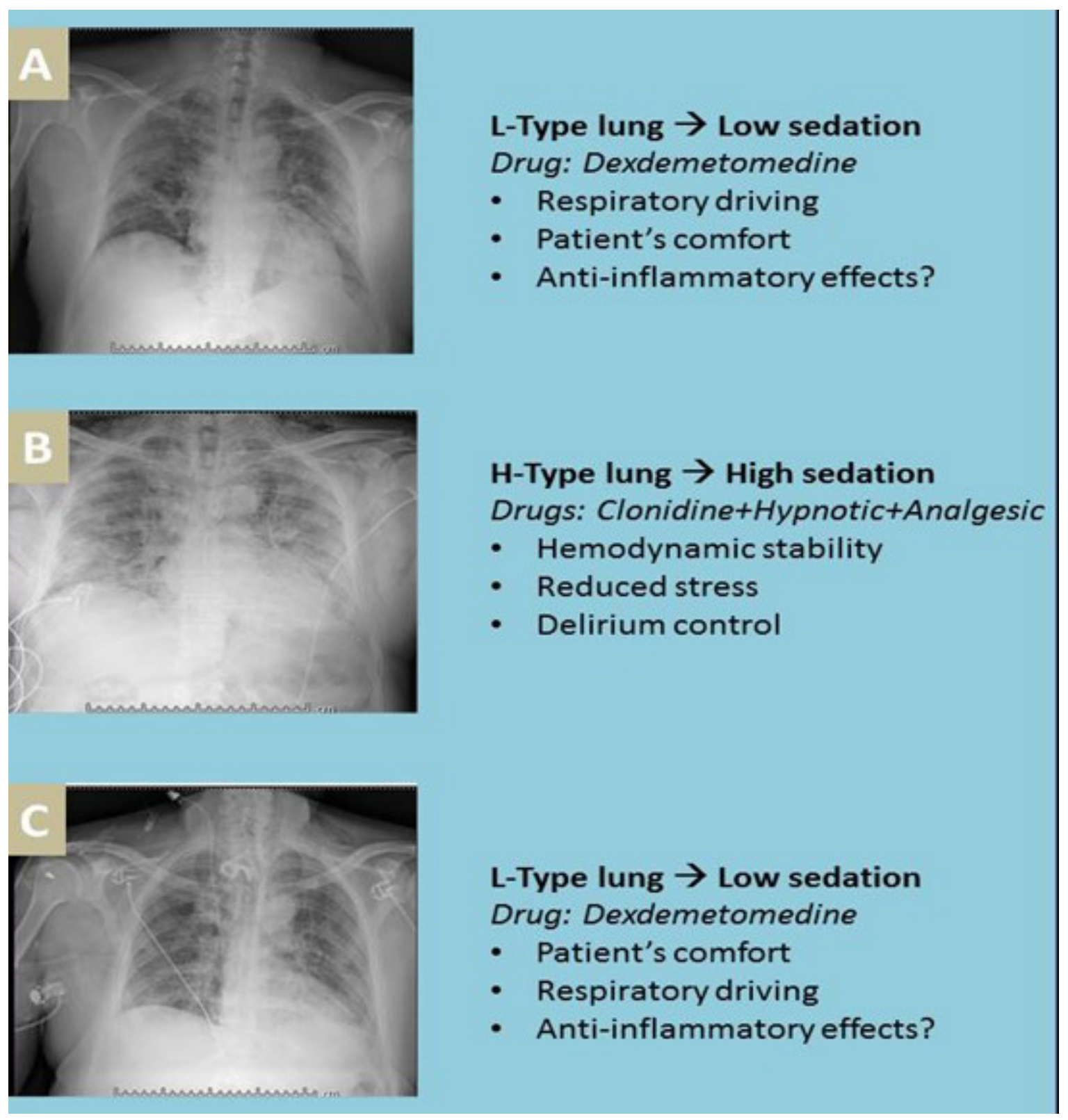

FIGURE 1. The three step sedation model. (A) The L-Type lung phenotype may require light sedation through dexmedetomidine for maintaining the respiratory drive and improve patient comfort. (B) A worsening in the respiratory picture (Type H) may require mechanical ventilation. It imposes deep sedation through hypnotic and analgesic medications. Compared to dexmedetomidine, clonidine has fewer sedative properties (600 times lower); it can reduce the hypnotic dose, providing better hemodynamic stability. (C) When respiratory performance improves, dexmedetomidine-based light sedation can reduce the time to extubation with beneficial effects on sedation-induced complications and potential anti-inflammatory effects.

\section{ACKNOWLEDGMENT}

The authors thank all the peer reviewers for their opinions and suggestions.

\section{FUNDING}

This study received no external funding.

\section{CONFLICT OF INTEREST}

The authors declare no conflict of interest. Marco Cascella is a member of the Editorial Board of this journal.

\section{REFERENCES}

[1] Gattinoni L, Chiumello D, Caironi P, Busana M, Romitti F, Brazzi L, et al. COVID-19 pneumonia: different respiratory treatments for different phenotypes? Intensive Care Medicine. 2020; 46: 1099-1102.

[2] Alhazzani W, Belley-Cote E, Møller MH, Angus DC, Papazian L, Arabi YM, et al. Neuromuscular blockade in patients with ARDS: a rapid practice guideline. Intensive Care Medicine. 2020; 46: 1977-1986.

[3] Chanques G, Constantin J, Devlin JW, Ely EW, Fraser GL, Gélinas C, et al. Analgesia and sedation in patients with ARDS. Intensive Care Medicine. 2020; 46: 2342-2356.

[4] Mohammadi M, Ahmadi M, Khalili H, Cheraghchi H, Arbabi M. Cyproheptadine for the Prevention of Postoperative Delirium. Annals of Pharmacotherapy. 2016; 50: 180-187.

[5] Lin OA, Karim ZA, Vemana HP, Espinosa EV, Khasawneh FT. The 
antidepressant 5-HT2a receptor antagonists Pizotifen and cyproheptadine inhibit serotonin-enhanced platelet function. PLoS ONE. 2014; 9: e87026.

[6] Devlin JW, Skrobik Y, Gélinas C, Needham DM, Slooter AJC, Pandharipande PP, et al. Clinical Practice Guidelines for the Prevention and Management of Pain, Agitation/Sedation, Delirium, Immobility, and Sleep Disruption in Adult Patients in the ICU. Critical Care Medicine. 2018; 46: e825-e873.

[7] Paternoster G, Sartini C, Pennacchio E, Lisanti F, Landoni G, Cabrini L. Awake pronation with helmet continuous positive airway pressure for COVID-19 acute respiratory distress syndrome patients outside the ICU: a case series. Medicina Intensiva. 2020; S0210-5691(20)30273-4.

[8] Mehta S, Spies C, Shehabi Y. Ten tips for ICU sedation. Intensive Care Medicine. 2018; 44: 1141-1143.

[9] Cloesmeijer ME, Oever HLA, Mathôt RAA, Zeeman M, Kruisdijk-Gerritsen A, Bles CMA, et al. Optimising the dose of clonidine to achieve sedation in intensive care unit patients with population pharmacokinetics. British Journal of Clinical Pharmacology. 2020; 86: $1620-1631$.

[10] Weerink MAS, Struys MMRF, Hannivoort LN, Barends CRM, Absalom AR, Colin P. Clinical Pharmacokinetics and Pharmacodynamics of Dexmedetomidine. Clinical Pharmacokinetics. 2017; 56: 893-913.

[11] Shafer SL, Hendrickx JFA, Flood P, Sonner J, Eger EI. Additivity versus synergy: a theoretical analysis of implications for anesthetic mechanisms. Anesthesia and Analgesia. 2008; 107: 507-524.

[12] Adams CD, Altshuler J, Barlow BL, Dixit D, Droege CA, Effendi MK, et al. Analgesia and Sedation Strategies in Mechanically Ventilated Adults with COVID-19. Pharmacotherapy. 2020; 40: 1180-1191.

[13] Buckley MS, Smithburger PL, Wong A, Fraser GL, Reade MC, Klein-Fedyshin M, et al. Dexmedetomidine for Facilitating Mechanical Ventilation Extubation in Difficult-to-Wean ICU Patients: Systematic
Review and Meta-Analysis of Clinical Trials. Journal of Intensive Care Medicine. 2020; 885066620937673.

[14] Jain A, Lamperti M, Doyle DJ. Dexmedetomidine: another arrow in the quiver to fight COVID-19 in intensive care units. British Journal of Anaesthesia. 2021; 126: e35-e38.

[15] Zhao H, Davies R, Ma D. Potential therapeutic value of dexmedetomidine in COVID-19 patients admitted to ICU. British Journal of Anaesthesia. 2021; 126: e33-e35.

[16] Cascella M, Mauro I, De Blasio E, Crispo A, Del Gaudio A, Bimonte $\mathrm{S}$, et al. Rapid and Impressive Response to a Combined Treatment with Single-Dose Tocilizumab and NIV in a Patient with COVID-19 Pneumonia/ARDS. Medicina. 2020; 56: 377.

[17] Cascella M, Fiore M, Leone S, Carbone D, Napoli RD. Current controversies and future perspectives on treatment of intensive care unit delirium in adults. World Journal of Critical Care Medicine. 2019; 8: 1827.

[18] Stockton J, Kyle-Sidell C. Dexmedetomidine and worsening hypoxemia in the setting of COVID-19: a case report. American Journal of Emergency Medicine. 2020; 38: 2247.e1-2247.e2.

[19] Czepiel KS, Lucas AT, Whalen MJ, Mojica JE. DexmedetomidineAssociated Hyperpyrexia in Three Critically Ill Patients with Coronavirus Disease 2019. Critical Care Explorations. 2020; 2: e0213.

How to cite this article: Alfredo Del Gaudio, Giuseppe Mincolelli, Andreaserena Recchia, Elvio De Blasio, Maura C Tracey, Marco Cascella. Tailored sedation in critically ill COVID-19 patients based on lung damage, and pharmacokinetic and pharmacodynamic profiles. Signa Vitae. 2021;17(6):157161. doi:10.22514/sv.2021.115. 